\title{
A Boussinesq type extension of the GeoClaw model - a study of wave breaking phenomena applying dispersive long wave models
}

\author{
Jihwan Kim $^{\mathrm{a}}$, Geir K. Pedersen ${ }^{\mathrm{a}}$, Finn Løvholt ${ }^{\mathrm{a}, \mathrm{b}}$, Randall J. LeVeque ${ }^{\mathrm{c}}$ \\ ${ }^{a}$ University of Oslo, Department of Mathematics, Oslo, Norway \\ ${ }^{b}$ Norwegian Geotechnical Institute, Oslo, Norway \\ ${ }^{c}$ University of Washington, Department of Applied Mathematics, Seattle, USA
}

\begin{abstract}
The nonlinear shallow water model is widely used in the study of tsunami propagation, but an increasing number of studies are dedicated to the dispersion dynamics of tsunamis. If the wave dispersion becomes important, Boussinesqtype models are often used. In this work, a general purpose Boussinesq solver, BoussClaW, is introduced for modeling non-linear dispersive tsunami propagation, taking into account inundation. The BoussCLAW model is an extension of the GeoClaw tsunami model. It employs a hybrid of finite volume and finite difference methods to solve Boussinesq equations from the literature, which are based on the depth-averaged velocity and include enhanced dispersion properties. On the other hand, in the selected formulation only some non-linearity is retained in the dispersion term. In order to validate BoussClaw, numerical results are compared to analytic solutions, solutions obtained by pre-existing models, and laboratory experiments. Even though the equations of BoussCLAW are not fully nonlinear they perform far better than standard Boussinesq equations with only linear dispersion terms. Furthermore, the wave steepening and breaking motion is carefully scrutinized, and we demonstrate that the point of wave breaking may be wrongly identified in many of the commonly used Boussinesq models.
\end{abstract}

Keywords: Breaking wave, Boussinesq equation, finite volume method 


\section{Introduction}

Tsunamis are generally long waves compared to the water depth, and longwave models are consequently widely used in the study of their propagation and inundation. Through the use of numerical shock capturing techniques for

5 modeling the near-shore bore formation of the tsunami, nonlinear shallow water (NLSW) models did become the standard model for modeling tsunami propagation and run-up, see e.g. (Titov and Synolakis, 1995; Imamura, 1996; Harig et al. 2008, Berger et al., 2011).

The NLSW models do not incorporate frequency dispersion, which may be included by ascending in the hierarchy of long wave expansion to Boussinesq type equations. Numerical models based on Boussinesq type equations have been used for idealized studies of wave processes since 1966 (Peregrine, 1966) and additionally to simpler problems in coastal engineering in the following decades (Brocchini, 2013). The accumulated effect of the frequency dispersion for the wave propagation over the open sea is a function of propagation time and the shape of the disturbance (Glimsdal et al. 2013), and may become important for some tsunamis, in particular for landslide sources (Løvholt et al. 2015). Dispersion may further be of importance, in combination with nonlinear effects, for the evolution of undular bores for tsunamis (Glimsdal et al. 20 2013, Grue et al. 2008, Løvholt et al., 2008, Behrens and Dias, 2015). In the last decades we have seen a development on long wave expansions and their numerical formulations. In the 1990s the modeling with Boussinesq type equations were vitalized by new formulations, in particular those of Madsen and Sørensen (1992) and Nwogu (1993) which displayed improved dispersion

25 properties in comparison to the standard formulation of Peregrine (1967). Later still more extensions and improvements have followed as described in the reviews Madsen et al. (2003), Brocchini (2013) and Kirby (2016).

Boussinesq-type equations differ in mathematical structure from the NLSW equations and do not inherit characteristics in the same simple form. Hence, 
breaking motion in Boussinesq models. Schäffer et al. (1993) employed the concept of the surface roller, first proposed by Svendsen (1984), which is a volume of water passively riding at the bore front. Tissier et al. (2012) suggested a breaking model based on the surface roller, the maximal front angle and the Froude number. Another way of incorporating breaking was suggested by Kennedy et al. (2000) who included diffusive terms in the momentum equation. These diffusive terms were activated and deactivated as a steepness measure crossed thresholds. The original steepness measure was the temporal rate of surface elevation corresponding to a very steep solitary wave. Later, Lynett (2006) investigated a variety of steepness measures and then identified that the surface steepness provides the least sensitive breaking threshold. Løvholt et al. (2013) similarly employed a diffusive model including transport terms, but pointed out that breaking wave Boussinesq models were prone to instabilities. An alternative non-linear diffusive ad-hoc breaking term was suggested by Matsuyama

45 et al. (2007), based on their large scale experiments of the wave propagation of undular bores on various slope angles.

Naturally, there is a desire to exploit the efficient and well established shock capturing framework of the NLSW models also in a dispersive context. Antuono et al. (2009) remolded the whole Boussinesq equations into a framework on 50 hyperbolic form. However, most of the recently developed Boussinesq models are based on some combination of approximate Riemann solvers, with TVD limiters, for the hydrostatic transport terms and finite differences for dispersion terms (Erduran et al., 2005, Kim et al., 2009, Shiach and Mingham, 2009, Roeber et al., 2010; Dutykh et al., 2011; Shi et al., 2012). Among other models, this has led to the popular Funwave-TVD and Coulwave-TVD applications. In most Boussinesq models that include runup on beaches, the dispersion term is turned off in the vicinity of the shoreline to avoid interference of the wettingdrying techniques with the larger computational stencils from the dispersion terms. Still, the dispersion terms are often seen to cause stability problems in ${ }_{60}$ the strongly nonlinear parts of the shoaling process (Løvholt et al., 2013). In fact, a practice of switching to the NLSW equations in the near-shore region, 
where large amplitude-to-depth-ratios occur, has evolved. This allows for a relatively robust treatment of the modeling of the post breaking phase. To this end, Tonelli and Petti (2009) and Shi et al. (2012), for instance, employ 65 a wave-height to depth threshold of 0.8 which is motivated by the maximum height of an undular bore, which again is related to the extreme solitary wave. This threshold is a pragmatic choice for gentle bottom gradients and may be questionable under other circumstances.

In this paper, we present a new hybrid Boussinesq type model BoussCLAw, of similar mold as Funwave-TVD and Coulwave-TVD, but with a different Boussinesq formulation. In particular, the dispersion term is simpler and not fully nonlinear, as robustness is given priority over high formal order. The goal of the present article is twofold. First, to present a careful validation of the BoussCLAW model, both towards laboratory experiments and reference models.

75 Second, we use the new model to explore the breaking phenomena in the context of Boussinesq equations. It is investigated how different Boussinesq type models can represent the wave evolution until the point of breaking. In the presented example, we are finally able to demonstrate that Boussinesq models may stably compute the near shore tsunami propagation beyond the standard 0.8 waveheight-to-depth threshold. Conversely, we find that the use of this threshold invokes a too early formation of a breaking bore. This points indicates that the breaking criteria employed so far lacks generality.

This paper is organized as follows: In Section 2 , the base model for the wave equations is given and the numerical scheme is outlined, while a von Neuman stability analysis is put in Appendix A. Sections 3 compares results from the Bouss CLAW with analytic ones, laboratory experiments and those from other models. In subsection 4.1 we scrutinize the pre-breaking shoaling of Boussinesq type equations through comparison with full potential theory, while the postbreaking evolution is investigated in subsection 4.2 . 


\section{Model Description}

Boussinesq-type equations are derived on the assumption that the ratio of depth to wavelength, $\mu$, is small. In addition one may assume that the ratio of wave amplitude to depth, $\epsilon$, is small. Different kinds of long wave assumptions are then generally characterized by relative errors in terms of these two parameters. Herein we will neither derive Boussinesq equation nor make the equations dimensionless as such. Still, $\mu$ and $\epsilon$ will sometimes be used to indicate relative errors. Moreover, when presenting results we will often use dimensionless quantities which are marked by a star. The horizontal and vertical and temporal coordinates are denoted by $x, y$ and $t$, respectively, while the depth averaged horizontal velocity and the surface elevation are denoted by $u$ and $\eta$, respectively. Dimensionless variables are then defined as

$$
t^{*}=\sqrt{\frac{g}{h_{0}}} t, \quad x^{*}=\frac{x}{h_{0}}, \quad \eta^{*}=\frac{\eta}{h_{0}}, \quad u^{*}=\frac{u}{\sqrt{g h_{0}}}, \quad \text { etc. }
$$

where $h_{0}$ is a reference depth which is chosen as the maximum equilibrium depth. Dimensional variables will be used in the sections 2 , 3.2 Appendix A Appendix B, and finally the figures 3, 4 and 5. Elsewhere, dimensionless variables are employed. Sometimes the dimensionless quantities are spelled out, such as $x / h_{0}$, but mostly starred quantities are used.

\subsection{BoussCLAW - a new long wave model for tsunami propagation and run-up}

In this work, a new numerical model, called BoussClaw, is introduced. It is an extension of GeoClaw (Clawpack Development Team, 2016), and solves the Boussinesq-type equations derived by Schäffer and Madsen (1995). The extended model is formulated in two horizontal directions, but herein we focus on the description of plane waves for simplicity. Tests and details on the performance with two horizontal directions are found in Kim (2014).

The Bouss Claw model employ a finite volume technique for the NLSW part of the equations and a finite difference discretization in fractional steps. 
2016) developed mainly by LeVeque (1997), George (2008) and Berger et al. (2011), which is designed to solve the nonlinear shallow water equations.

\subsubsection{Boussinesq-type equations}

Schäffer and Madsen (1995) derived Boussinesq-type equations where addition of a higher order $O\left(\mu^{4}\right)$ term enabled optimization of linear dispersion properties. We restrict ourselves to the choice $B_{2}=0$ from the formulation of Schäffer and Madsen (1995). The equations then read

$H_{t}+(H u)_{x}=0$,

$(1-D)\left[(H u)_{t}\right]+\left(H u^{2}+\frac{g}{2} H^{2}\right)_{x}-g H h_{x}-B g h^{2}\left(h \eta_{x}\right)_{x x}=-f_{D}$,

where we have added a Manning type friction term, denoted by $f_{D}$ and defined in eq. 122 The operator $D$ is defined in terms of the dummy variable $w$ according to

$D(w)=\left(B+\frac{1}{2}\right) h^{2} w_{x x}-\frac{1}{6} h^{3}\left(\frac{w}{h}\right)_{x x}$,

for any $w(x, t)$. In the above equations $H(x, t)$ and $u(x, t)$ are the total flow

depth and the depth averaged velocity of the water, respectively, $h(x)$ is the still water depth, $\eta(x, t)$ is the surface elevation, and thus $H(x, t)=h(x)+\eta(x, t)$. Moreover, $g$ is the acceleration of gravity, and $B$ is a dispersion parameter. Madsen and Sørensen (1992) have chosen $B=1 / 15$ for which the dispersion relation from the Boussinesq equations follows linear potential theory to a higher

order in wave number times depth. When $B=0$, this set of the Boussinesq-type equations approximately reduces to that of Peregrine (1967) as the linear dispersion relations are identical. However, unlike Peregrine's momentum equation the hydrostatic parts of (3) are written in a conservative form. Moreover, some nonlinearity is introduced in the dispersion term. Even though (2), (3) and (4) do not constitute a fully nonlinear set of Boussinesq equations, inheriting relative errors of order $\mu^{2}, \epsilon \mu^{2}$, they do describe shoaling of solitary waves markedly better than, for instance, the Peregrine equations, as will be demonstrated in section 4.1 
The Bouss Claw model solves the Boussinesq-type equations (2) and (3) numerically with a hybrid combination of the finite volume and finite difference methods that will be explained in a moment. There have been several studies of this type of hybrid schemes. For example, see Tissier et al. (2011), Shi et al. (2012) and Dutykh et al. (2013).

To facilitate a fractional step method, as outlined below, we move the hydrostatic terms of $(3)$ inside the $(1-D)$ operator, while balancing with extra terms in the $\Psi$, to obtain

$(1-D)\left[(H u)_{t}+\left(H u^{2}+\frac{g}{2} H^{2}\right)_{x}-g H h_{x}\right]=-\Psi(x, t)-f_{D}$,

where

$$
\begin{aligned}
\Psi(x, t)= & \left(B+\frac{1}{2}\right) h^{2}\left(\left(H u^{2}\right)_{x}+g H \eta_{x}\right)_{x x} \\
& -\frac{1}{6} h^{3}\left(\frac{\left(H u^{2}\right)_{x}+g H \eta_{x}}{h}\right)_{x x}-B g h^{2}\left(h \eta_{x}\right)_{x x} .
\end{aligned}
$$

\subsubsection{Numerical scheme}

The equations (2) and (5) are written in a conservative form with respect to the leading order terms in $\mu$, but with the $\Psi$ term as a pseudo source. Such equations may be solved by a fractional step method as described in LeVeque (2002). First, it is observed that (5) may be formally rewritten as

$$
(H u)_{t}=-\left\{\left(H u^{2}+\frac{g}{2} H^{2}\right)_{x}-g H h_{x}\right\}-(1-D)^{-1} \Psi(x, t)-(1-D)^{-1} f_{D},
$$

At the first stage of the hybrid scheme, we integrate $H u$ over a time step taking into account all hydrostatic terms, namely those within the braces on the right hand side, and omitting the source terms involving $\Psi$. When this is combined with the continuity equation (2) this simply corresponds to advancing the shallow water equations one time step forward. To this end we employ GEOCLAW, a high-order accurate finite volume solver for the shallow water equations.

Next, the Manning resistance term is accounted for. To this end we ignore the coupling of bottom friction and dispersion (replace $(1-D)^{-1}$ by 1 in $(7)$ ) and employ the semi-implicit solver in Geoclaw for $(H u)_{t}=-f_{D}$. 
In the final stage, we retain the $H$ value, but integrate $H u$ (essentially being the momentum density) further from the two first stages by solving

$$
(1-D)\left[(H u)_{t}\right]=-\Psi
$$

Since the differential operator $D$ contains spatial derivatives, a systems of difference equations must then be solved.

The spatial and time discretization should be carefully chosen for the stability of the second stage. In our numerical scheme, the second order centered scheme is used for the spatial discretization, and a four stage Runge-Kutta method is used for the time integration. The von Neumann stability analysis of this numerical scheme is outlined in Appendix A.

Suppose the spatial domain is divided into $n$ grid cells with the spatial grid size $\Delta x$. Arrays of nodal values for flow depth and $H u$, respectively, are defined as

$$
\begin{aligned}
\mathbf{H} & =\left(H_{1}, H_{2}, \ldots, H_{n}\right)^{T}, \\
\mathbf{M} & =\left(H_{1} u_{1}, H_{2} u_{2}, \ldots, H_{n} u_{n}\right)^{T} .
\end{aligned}
$$

With time increment $\Delta t$ the fourth order Runge-Kutta scheme can be written as follows,

$$
\mathbf{M}^{1}=\mathbf{M}, \quad \mathbf{M}^{2}=\mathbf{M}+\frac{\Delta t}{2} \mathbf{S}^{1}, \quad \mathbf{M}^{3}=\mathbf{M}+\frac{\Delta t}{2} \mathbf{S}^{2}, \quad \mathbf{M}^{4}=\mathbf{M}+\Delta t \mathbf{S}^{3},
$$

where $\mathbf{M}^{k}$ are intermediate value arrays and $\mathbf{S}^{k}$ are correspondingly arrays for the time derivatives of $\mathrm{Hu}$, obtained by solving

$(I-\bar{D}) \mathbf{S}^{k}=-\bar{\Psi}\left(\mathbf{H}, \mathbf{M}^{k}\right), \quad$ for $k=1, \ldots, 4$.

Here $\bar{\Psi}$ and $\bar{D}$ represent centered spatial discretization for the term $\Psi$ and the operator $D$, respectively. These are given explicitly below. Finally the value of $\mathbf{M}$ at the new time level is obtained by

$$
\mathbf{M}^{+}=\mathbf{M}+\frac{\Delta t}{6}\left[\mathbf{S}^{1}+2 \mathbf{S}^{2}+2 \mathbf{S}^{3}+\mathbf{S}^{4}\right] .
$$


In $10, \bar{D}$ is a tri-diagonal $n \times n$ matrix with elements

$$
\begin{aligned}
\bar{D}_{i, i-1} & =\frac{1}{\Delta x^{2}}\left[\left(B+\frac{1}{2}\right) h_{i}^{2}-\frac{1}{6} \frac{h_{i}^{3}}{h_{i-1}}\right], \\
\bar{D}_{i, i} & =\frac{1}{\Delta x^{2}}\left(-2 B-\frac{2}{3}\right) h_{i}^{2}, \\
\bar{D}_{i, i+1} & =\frac{1}{\Delta x^{2}}\left[\left(B+\frac{1}{2}\right) h_{i}^{2}-\frac{1}{6} \frac{h_{i}^{3}}{h_{i+1}}\right] .
\end{aligned}
$$

Correspondingly, the $i$-th element of $\Psi(\overline{\mathbf{H}}, \mathbf{q})$ is

$$
\begin{aligned}
\bar{\Psi}_{i} & =\left(B+\frac{1}{2}\right) \frac{h_{i}^{2}}{2 \Delta x^{3}}\left[\left(\frac{M_{i+2}^{2}}{H_{i+2}}-2 \frac{M_{i+1}^{2}}{H_{i+1}}+2 \frac{M_{i-1}^{2}}{H_{i-1}}-\frac{M_{i-2}^{2}}{H_{i-2}}\right)\right. \\
& \left.+g\left(H_{i+1}\left(\eta_{i+2}-\eta_{i}\right)-2 H_{i}\left(\eta_{i+1}-\eta_{i-1}\right)+H_{i-1}\left(\eta_{i}-\eta_{i-2}\right)\right)\right] \\
& -\frac{1}{6} \frac{h_{i}^{3}}{2 \Delta x^{3}}\left[\frac{M_{i+2}^{2} / H_{i+2}-M_{i}^{2} / H_{i}}{H_{i+1}}-2 \frac{M_{i+1}^{2} / H_{i+1}-M_{i-1}^{2} / H_{i-1}}{h_{i}}\right. \\
& +\frac{M_{i}^{2} / H_{i}-M_{i-2}^{2} / H_{i-2}}{H_{i-1}} \\
& \left.+g\left(\frac{H_{i+1}\left(\eta_{i+2}-\eta_{i}\right)}{H_{i+1}}-2 \frac{H_{i}\left(\eta_{i+1}-\eta_{i-1}\right)}{h_{i}}+\frac{H_{i-1}\left(\eta_{i}-\eta_{i-2}\right)}{H_{i-1}}\right)\right] \\
& -\frac{B g h_{i}^{2}}{2 \Delta x^{3}}\left(H_{i+1}\left(\eta_{i+2}-\eta_{i}\right)-2 H_{i}\left(\eta_{i+1}-\eta_{i-1}\right)+H_{i-1}\left(\eta_{i}-\eta_{i-2}\right)\right),
\end{aligned}
$$

for $i=1,2, \ldots, n$.

\subsubsection{Additional numerical features}

Following Tonelli and Petti (2009) and Shi et al. (2012) we may represent wave breaking in a heuristic fashion through invocation of a threshold $\epsilon_{B}:=$ $\eta / h=0.8$ in BoussClaw . When the threshold is reached, the wave breaking is supposed to be initiated, and the dispersive terms are suppressed throughout the computational domain. This is adequate for the applications presented herein, which involve a single solitary wave. For other applications the switch to the NLSW equations should be made for only a subregion of the computational domain.

Bottom friction is important for inundation on gentle slopes. Figure 10 exemplifies that the friction reduces the run-up height. BoussClaw uses the 
Manning-type friction as follows,

$$
f_{D}=-\frac{g C_{d}^{2} u|u|}{H^{5 / 3}}
$$

In Section 3, a non-dimensional Manning friction coefficient is used as $C_{d}^{*}=$

$C_{d} \sqrt{\frac{g}{h_{0}}} h_{0}^{-1 / 3}$, with the typical range between 0.01 and 0.04 (Chow, 1959).

In the wetting-drying process during runup a parameter for the dry tolerance $d_{t o l}$ is chosen such that $H$ is put to 0 if $H<d_{t o l} h_{0}$, where $h_{0}$ is the reference depth (maximum equilibrium depth). In this work, the parameter $d_{t o l}$ is set to $10^{-4}$. The dispersion terms of the Boussinesq equations imply an extended computational stencil as compared to that for the NLSW equations. To prevent this stencil from including dry nodes and thereby produce irregularities and even instabilities, the dispersion terms are switched off when $h<N d_{t o l} h_{0}$ (close to the equilibrium shoreline), where the number $N$ is set to 100 for the simulations herein. Accordingly, for the dry land inundation, the Bouss Claw invariably utilizes the NLSW solver of the GEOCLAW software, which can handle wet and dry states with the depth positivity property. Details can be found in George (2008). The near-shore truncation of the dispersive terms produce small wriggles

(see figure6, right panel) that remain small as $\Delta x$ is reduced and do not influence the overall solution.

\subsection{Models for comparison}

The performance of the Boussinesq model presented here is partly assessed by comparison with numerical results from a full potential flow model which is described in Løvholt et al. (2013) and references therein. The model is based on a boundary integral technique and is run with fully nonlinear solitary wave solutions as initial conditions. During shoaling and breaking this model can describe the evolution of a plunger, but breaks down when the plunger reaches the free surface. Hence, the potential flow results are used to determine the point of breaking due to shoaling and to evaluate the evolution of amplitude and wave shape of the current model until this point. Below we refer to the full potential model as the BIM (Boundary Integral Method) model. 
Comparison with a pre-existing, fully nonlinear Boussinesq model is facilitated by the application of a Lagrangian model, described in Løvholt et al.

(2013). Apart from the use of Lagrangian coordinates the equations employed in this model are similar to (2) and (3). They differ only in the nonlinearities in the dispersion terms and in that the dispersion optimization terms are added in a fully nonlinear fashion. Presently, the Lagrangian model has no established bore capturing facility and is hence valid only to the point of breaking. Results from this model will be referred to as Serre, even though the dispersion enhancement is invoked.

Results for the Peregrine-type equations are obtained by the GloBouss model. This is a model for oceanic tsunami propagation which is based on discretization on a staggered grid. Further details are found in Løvholt et al. (2008).

For comparison also the version 2.1 of the FunwAVE-TVD model by Shi et al. (2012) is used. The FunWAVE-TVD model shares important features with BoussCLAW, employing a hybrid of the finite volume and finite difference scheme to solve the fully non-linear higher order dispersive Boussinesq model numerically. While we refer to Shi et al. (2012) for details, we briefly note that FunwaVE-TVD is based on the fully nonlinear Boussinesq equations of Chen (2006). The numerical spatial representation in FunwAVE-TVD is MUSCL TVD scheme to discretize for the flux and first order terms, whereas a central finite difference scheme (Wei et al., 1995a) is utilized for the higher order momentum terms. A Runge-Kutta scheme is employed for the time stepping.

\section{Comparing BoussClaw simulations with well-defined tests}

Four different test of BoussCLAw is presented. In Section 3.1 solitary wave propagation is considered. Even if this test is simple it includes dispersion and nonlinearity. Moreover, a scrutiny of the variation of energy with time and resolution shows that the artificial dissipation induced by the TVD part of the numerical scheme is very small for smooth waves. Solitary wave propagation in a moderately complex bathymetry is computed in section 3.2 and compared with 
experiments. Finally, more detailed studies of shoaling and runup properties of the model concern non-breaking (section 3.3.1) and breaking (section 3.3.2 runup. Together these tests should provide a solid assessment of BoussClaw. The results in the sections 3.1 and 3.3 are mainly presented in normalized coordinates, $t^{*}$ and $x^{*}$, as defined by (1), whereas results in section 3.2 are more conveniently expressed with dimensions retained.

\subsection{Solitary wave propagation}

For validation of the numerical approach solitary wave propagation is computed in constant water depth. For the initial conditions, the analytic solitary wave solution of the Serre's equations is used since exact analytic solutions are unknown for the set (2) and (3). In dimensionless coordinates the solitary wave solution of Serre's equations is given as

$$
\eta^{*}\left(x^{*}, t^{*}\right)=\alpha \operatorname{sech}^{2}\left(\kappa\left(x^{*}+c t^{*}-x_{i}^{*}\right)\right), \quad \text { and } \quad u^{*}=-c \frac{\eta^{*}}{1+\eta^{*}},
$$

where $x_{i}^{*}$ is the initial location of the crest peak, and

$\kappa=\sqrt{\frac{3 \alpha}{4(\alpha+1)}}, \quad$ and $\quad c=\sqrt{1+\alpha}$.

In these expressions $h_{0}$ is the equilibrium depth and $\alpha$ is the dimensionless amplitude. For the Serre model with enhanced dispersion we employ a modified version of 13 while the BIM model is initiated with Tanaka's solution. Details are given in Løvholt et al. (2013).

In Figure 1 surfaces from a BoussClaw simulation with $\Delta x^{*}=\Delta x / h_{0}=$ 0.05 are shown for amplitude $\alpha=0.2$. The computational results are in good agreement with the analytic solutions concerning height, shape and propagation speed. The amplitude decreases very gently as the wave propagates.

The integrated wave energies (per width) for the NLSW and Boussinesq equations are $E_{0}$ and $E_{0}+E_{1}$, respectively, as described in Appendix B. These quantities are made dimensionless by $E_{c}=\rho g h_{0}^{3}$, which is minus two times the equilibrium potential energy per width. In Figure $2 \mathrm{a}$ the time evolution of these energies are shown for $\alpha=0.2$ and $\Delta x^{*}=0.2$. There are tiny fluctuations both 


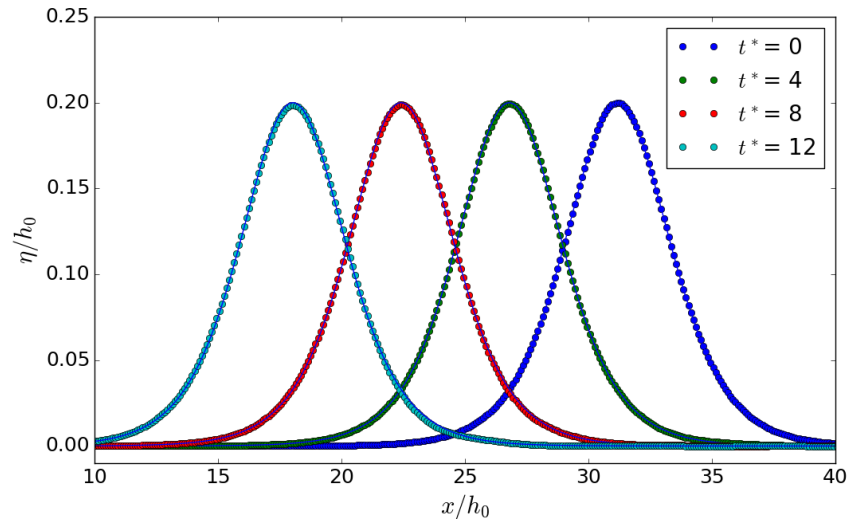

Figure 1: Analytic and computed solitary wave surfaces for $\alpha=0.2$ and $\Delta x^{*}=0.1$. The curves are marked by the normalized time $t^{*}$. The wave propagates from right to left, and the analytic solutions are solid lines.
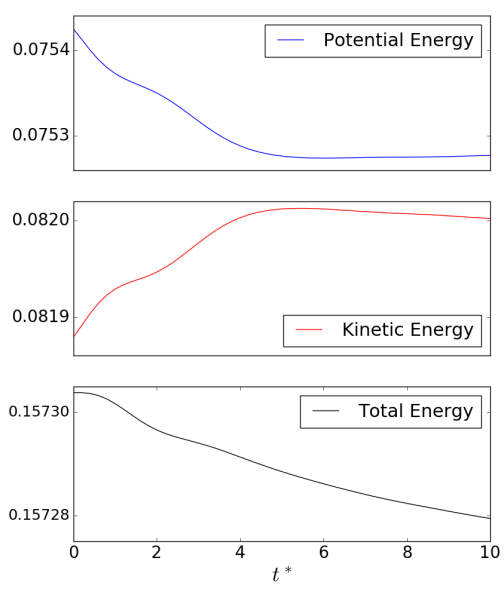

(a)

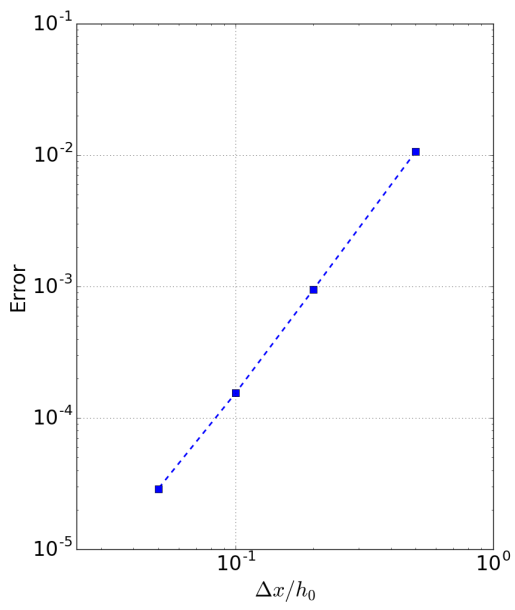

(b)

Figure 2: Evolution of energies for a solitary wave with $\alpha=0.2$. (a): Different parts of the dimensionless energy $E^{*}=E / E_{c}$. for $\Delta x^{*}=0.1$. (b): log-log plot of relative error of energy at $t^{*}=10$ for $\Delta x^{*}=0.05,0.1,0.2$ and 0.5 .

in the potential and kinetic energy that is evident when we zoom in, and the total energy decrease shows that the numerical procedure has a slight dissipation. In 
Figure $2 \mathrm{~b}$, the relative error of the energy at $t^{*}=10$,

$$
\text { Error }=\frac{\left|E_{t^{*}=0}^{*}-E_{t^{*}=10}^{*}\right|}{\left|E_{t^{*}=0}^{*}\right|},
$$

is shown for different $\Delta x^{*}$. For a solitary wave on a constant depth, the energy dissipation decreases with the grid increments.

\subsection{Waves on a composite slope}

A physical model was constructed at the Coastal Hydraulic Laboratory of the U.S. Army Corps of Engineers in order to address beach erosion and severe flooding problems (Briggs et al. 1995). The model beach consisted of three piece-wise linear slopes of 1:53, 1:150, and 1:13 with a vertical wall at the shoreline as shown in Figure 3. In the laboratory, the wave maker was located at $23.23 \mathrm{~m}$ from vertical wall and produced incident waves that were close to solitary waves. The gauge data from three cases are provided where the relative amplitude $\alpha$ equals $0.038,0.259$ and 0.681 , respectively, where $h_{0}=21.8 \mathrm{~cm}$ is the depth at the wave maker.

\begin{tabular}{|c|c|c|c|}
\hline \multicolumn{4}{|c|}{$23.23 \mathrm{~m}$} \\
\hline $15.04 \mathrm{~m}$ & \rfloor$\left.^{4} .36 \mathrm{~m}\right\rfloor$ & $2.93 \mathrm{~m}$ & $\jmath^{0.90 \mathrm{~m}}$ \\
\hline
\end{tabular}

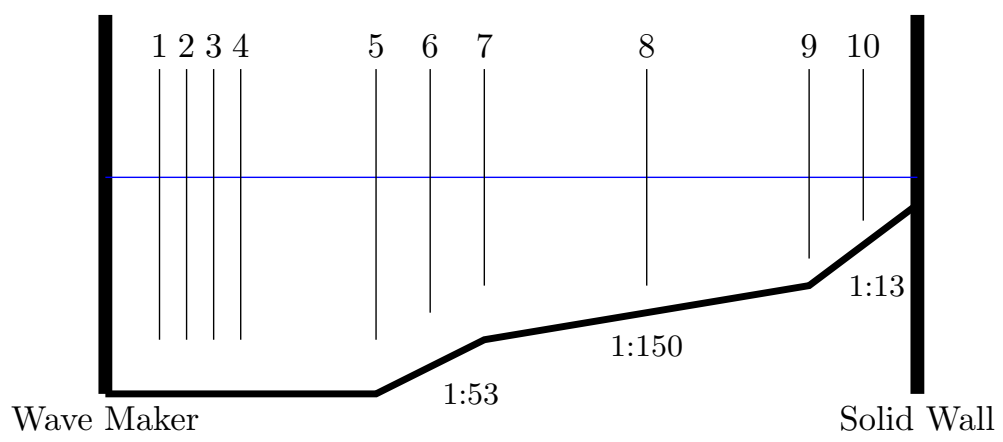

Figure 3: A sketch of the water tank used by Briggs et al. (1995).

For the second case, $\alpha=0.259$, numerical results have been compared to experiments with 400 grid points on a computational domain of $[-0.98,8.19]$ 
To specify the incident wave in BoussClaw, data from Gauge 4 were used for the wave height, while the second relation in (13) was used to obtain the corresponding velocity.

In Figure 4 water surface elevations at gauges 5, 7 and 8 are shown. The simulated waves are in good agreement with the laboratory measurements. For the reflected waves, larger discrepancies are observed. The increased discrepancy occurs because the full interaction between the wave and the wall at the right boundary is less accurately captured. Presumably, viscosity influences the wave evolution along the shallow region near the right wall, but we have not included these in the present numerical simulation. A better fit may possibly be obtained by incorporating a bottom friction.

\subsection{Shoaling and run-up of solitary waves}

Figure 5 shows the initial set-up for a test which follows the laboratory experiments by Synolakis (1987). The bathymetry of the wave tank is composed of a horizontal bottom, where the equilibrium depth is $h_{0}=0.196 \mathrm{~m}$, and a uniform slope as shown in Figure 5. A solitary wave of height $A_{0}$, hence $\alpha=$ $A_{0} / h_{0}$, is generated at the right end of the tank and propagates leftwards to the beach.

In the present section and throughout section 4 we present the results using the non-dimensional coordinates $\left(t^{*}, x^{*}\right)$, as defined by (1) with $h_{0}$ as the equilibrium depth in the flat bottom region In Synolakis (1987), $t^{*}=0$ was defined as when the wave crest was a non-dimensional distance, $L^{*}$, from the toe of the slope, where

$L^{*}=\sqrt{\frac{4}{3 \alpha}} \operatorname{arccosh}\left(\frac{1}{0.05}\right)$.

However, at $t^{*}=0$, the solitary wave has an elevation of $5 \%$ of it maximum at the toe of the beach, meaning that the slope has started to interact with the solitary wave. To avoid any such interaction obscuring our analysis, we instead place the initial solitary wave using equation 13 with $x_{i}^{*}=L^{*}+5 c$. In this way, 

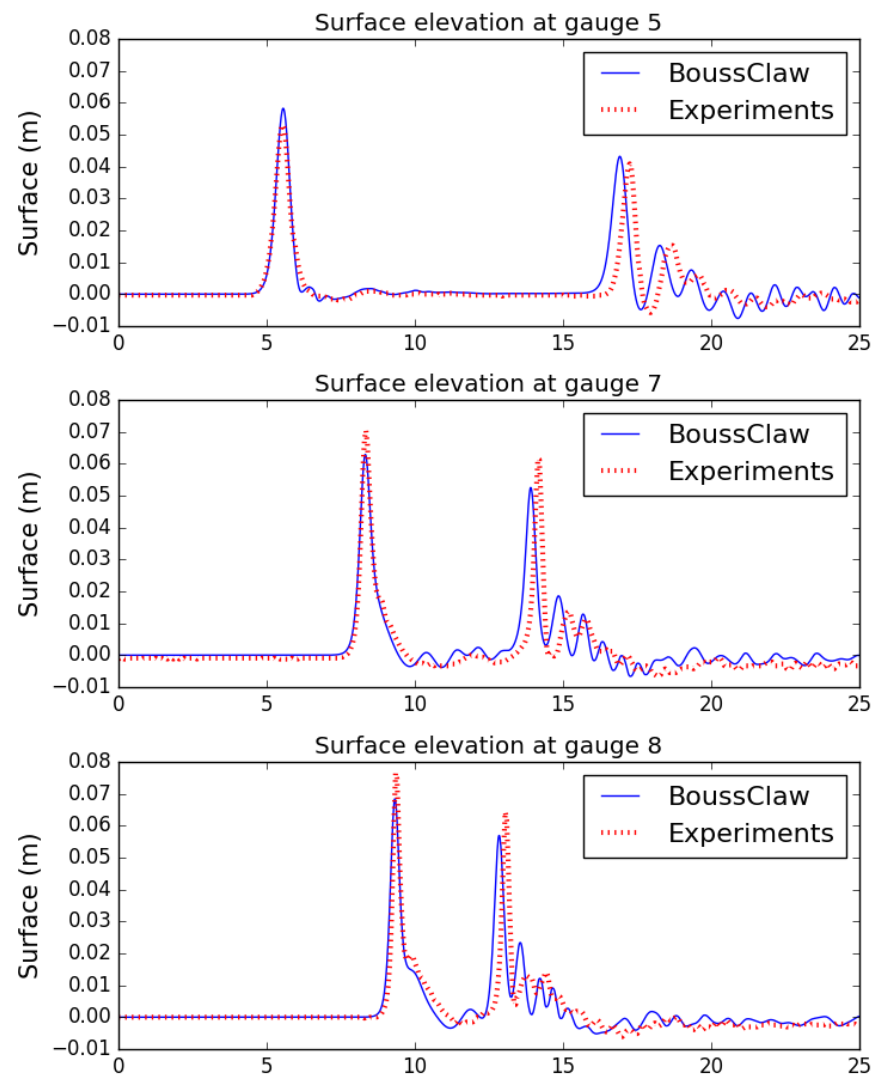

Figure 4: Comparison of BoussClaw and experiment from Briggs et al. (1995). Water surface elevation (m) in time (s) at gauges 5,7 and 8 for the $\alpha=0.259$ case.

an incident solitary wave of amplitude $\alpha \approx 0.3$, say, has a negligible interaction with the slope when initialized.

\subsubsection{Run-up of a non-breaking wave on a steep slope}

On a $10^{\circ}$ slope an incident solitary wave of amplitude $\alpha=0.3$ will not break until the end of the draw-down phase (Grilli et al. 1997). Still, this may be a challenging task for Boussinesq type models (Løvholt et al. 2013). Run-up on a $10^{\circ}$ slope was investigated experimentally by Pedersen et al. (2013) who found a theoretical overshoot of roughly $20 \%$ in the maximum run-up height. This was allotted to the viscous boundary layer on the beach and capillary effects. 


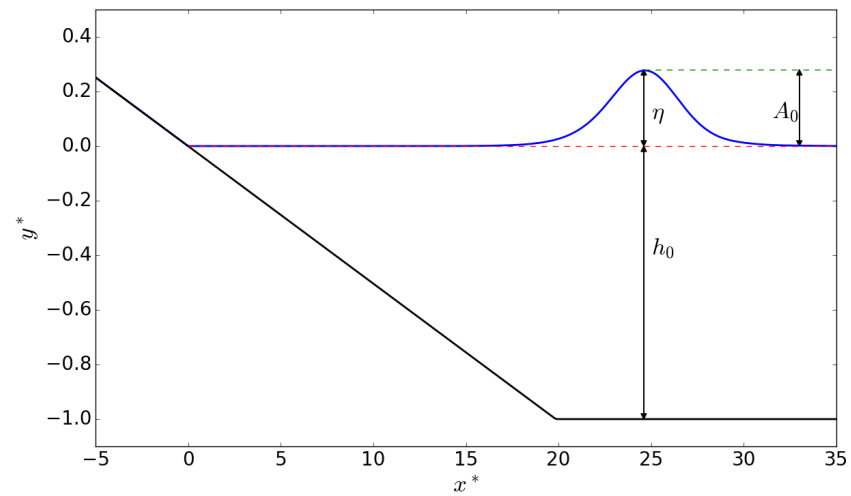

Figure 5: Definition sketch for shoaling and runup of solitary waves. The scale on the axes is the equilibrium depth, $h_{0}$.

Moreover, the measurements showed that the boundary layer flow during run-up was mostly laminar, albeit indications of transition was observed in the upper part of the swash tongue close to flow reversal. Hence, it is not appropriate to 275 employ a Manning friction term and we compare the models without any bed friction, while leaving the experiments out.
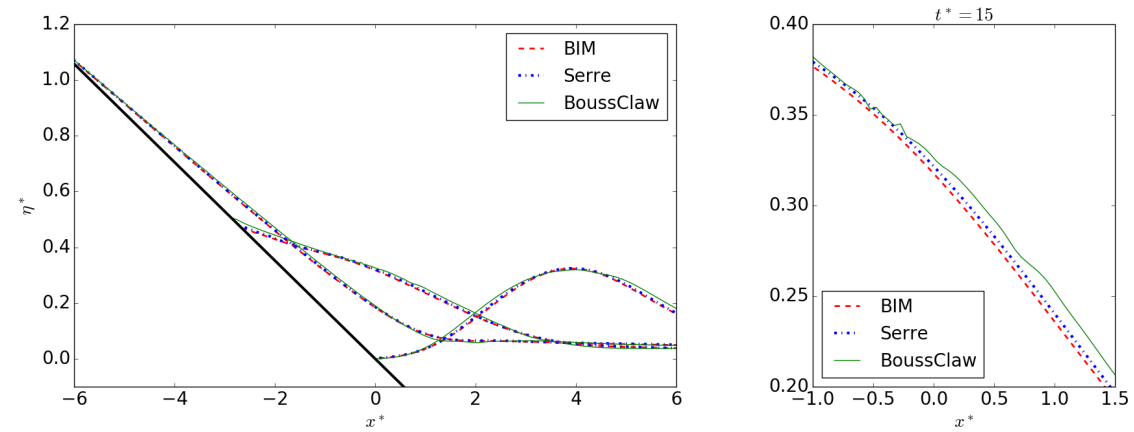

Figure 6: Runup of non-breaking solitary wave $\left(\alpha=0.3\right.$ and $\left.\theta=10^{\circ}\right)$. Left panel displays surfaces from the BIM, Serre and BoussClaw models at $t^{*}=10,15,20$ for $\Delta x^{*}=0.05$. Right figure is a zoom of the results at $t^{*}=15$

In Figure 6, the numerical results from BIM, Serre, and BoussClaw are shown at $t^{*}=10,15$, and 20 , and a zoom at $t^{*}=15$ is shown in the right panel. 
The agreement between the dispersive models are very good. Even though the

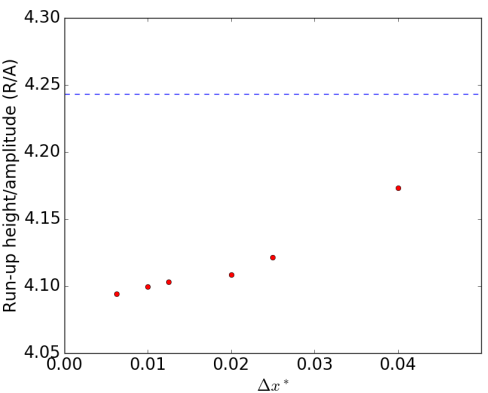

Figure 7: Non-breaking solitary wave $(\alpha=$ 0.3 and $\theta=10^{\circ}$ ). Maximum run-up divided by amplitude $\left(R^{*} / \alpha\right)$ from BoussCLAW with different grid sizes. The dashed line is from BIM. The grid size $\Delta x^{*}$ is $0.04 / \mathrm{N}$ and $0.025 / \mathrm{N}$ for $N=1,2,4$

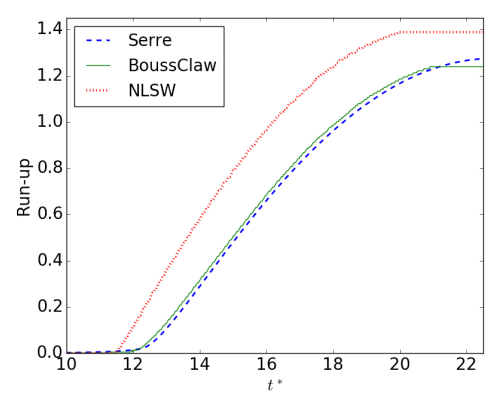

Figure 8: Non-breaking solitary wave $(\alpha=$ 0.3 and $\theta=10^{\circ}$ ). Time series for the runup height from Serre, BoussClaw and NLSW with $\Delta x^{*}=0.05$.

\subsubsection{Comparison with experiments on a breaking wave}

From the experiments of Synolakis (1987) on runup of solitary waves on beaches, we select the breaking case $\alpha=0.28$ incident on a $1: 19.85$ slope 


\begin{tabular}{c|cccc}
\hline Model & BIM & Serre & BoussClaw & NLSW \\
\hline Max. Run-up/Amp. & 4.2432 & 4.2488 & 4.0941 & 4.6561 \\
\hline
\end{tabular}

Table 1: Maximum run-up height divided by the incoming wave amplitude $\left(R^{*} / \alpha\right)$ for $\alpha=0.3$ and $\theta=10^{\circ}$.

for comparison with the BoussCLAW model. Experimental date is obtained at Synolakis et al. (2008).

In Figure 9, the laboratory measurements are shown with the computational results from the BoussClaw (in Boussinesq and NLSW mode), the Serre and the BIM models for $\alpha=0.28$ and a $1: 19.85$ slope at $t^{*}=15$. The grid size $\Delta x^{*}$ is 0.05 in the following simulations unless otherwise is stated. This is before the wave breaks and the BoussClaw, the Serre, and the BIM model are all in good agreement with the experiments.

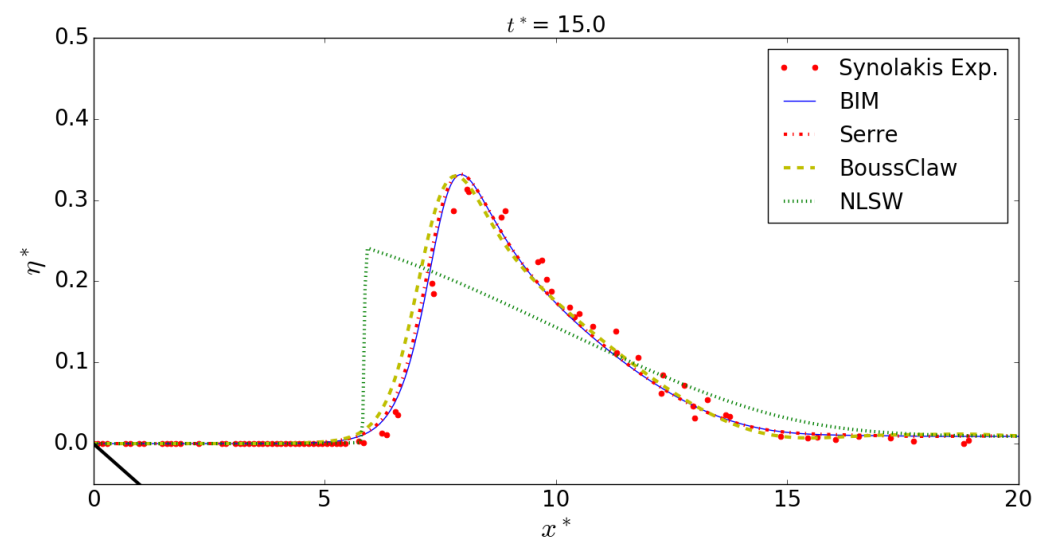

Figure 9: The surface elevation, $\eta^{*}$ as function of $x^{*}$ at $t^{*}=15$ from the laboratory experiments (Synolakis, 1987) ( $\alpha=0.28$, slope $1: 19.85)$, the BIM, the Serre, the BoussClaw and the NLSW models.

The ratio of amplitude to depth, $A^{*} / h^{*}\left(A^{*}\right.$ is the maximum value of $\eta^{*}$ and $h^{*}$ is the equilibrium depth at the corresponding location), is about 2 at the point of breaking. The potential flow model cannot be run much beyond the breaking points (until the attachment of the plunger only) and gives no 
information on the following bore propagation. In figure 10 we have compared the experimental data with the Bouss CLAW model of $C_{d}^{*}=0$ and 0.03 .

The agreement is good and the introduction of bed-friction even seem to match the truncated swash tongue of the experiments well. However, this may be a coincidence. Even though the wave has broken and some irregular flow features are introduced thereby, we have no evidence of the flow state being anywhere near turbulent, which is required for a quadratic bottom resistance to be appropriate. Capillary effects and experimental errors may also affect the comparison as observed by Pedersen et al. (2013).
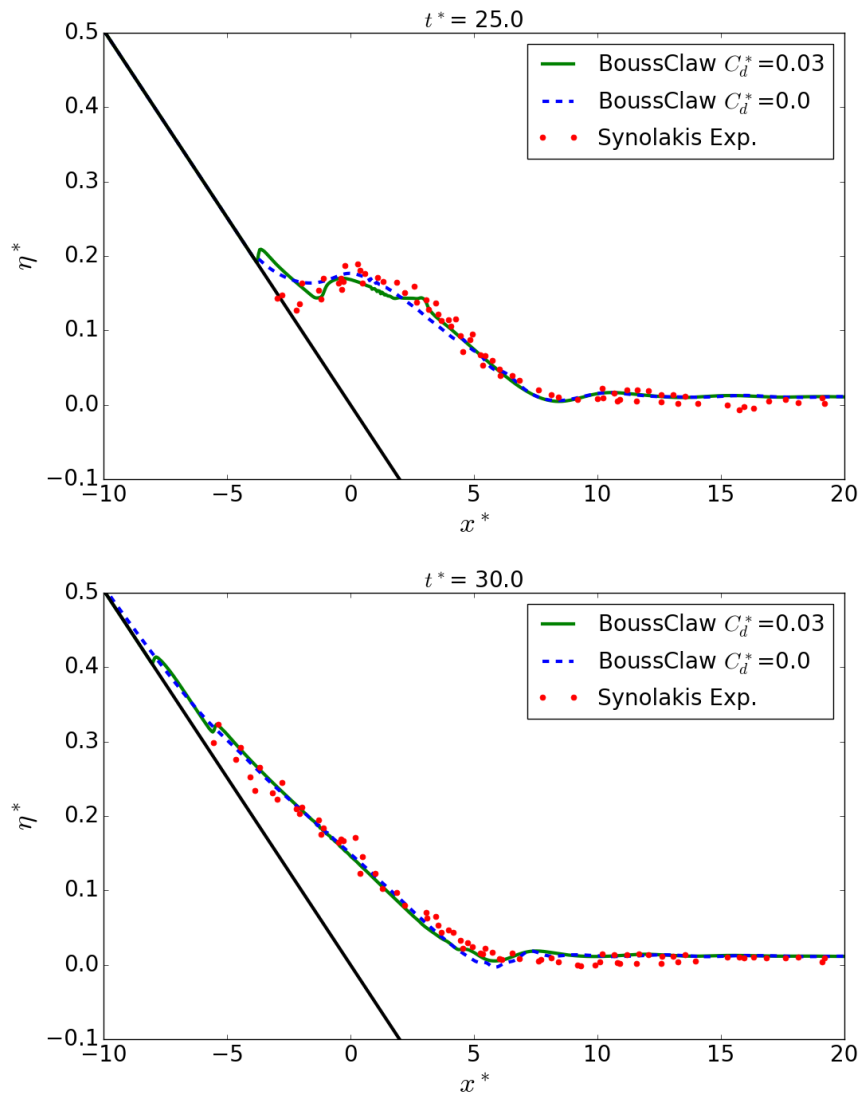

Figure 10: The surface elevation $\eta^{*}$ as function of $x^{*}$ at $t^{*}=25,30$ for the breaking case ( $\alpha=0.28$ and a $1: 19.85$ slope). Experiments and BoussClaw results with and without bottom drag are included. The resolution in the model is $\Delta x^{*}=0.05$. 
In Figure 11 and Table 2, we show the run-up height in time and the maximum run-up height respectively. Unlike what was observed for $\theta=10^{\circ}$, the NLSW model reduces the run up height. The opposite behavior for the two may be explained by two competing effects of dispersion. First, for a non-breaking wave the omission of non-hydrostatic effects lead to an excessive steepening of the wave front which implies higher run-up. On the other hand, the premature breaking dissipates energy and will reduce run-up heights. For the steeper slope, there is insufficient time for the second effect to fully counterbalance the first. For the gentler slope the early onset of breaking in the NLSW model, at a long distance form the shoreline, causes a large dissipation which dominates over the first effects.

In addition to affecting the runup a non-zero $C_{d}$ will delay, or even inhibit the withdrawal. A detailed discussion of such effects is outside the scope of the present article and we refer instead to a profound investigation in Antuono et al. (2012).

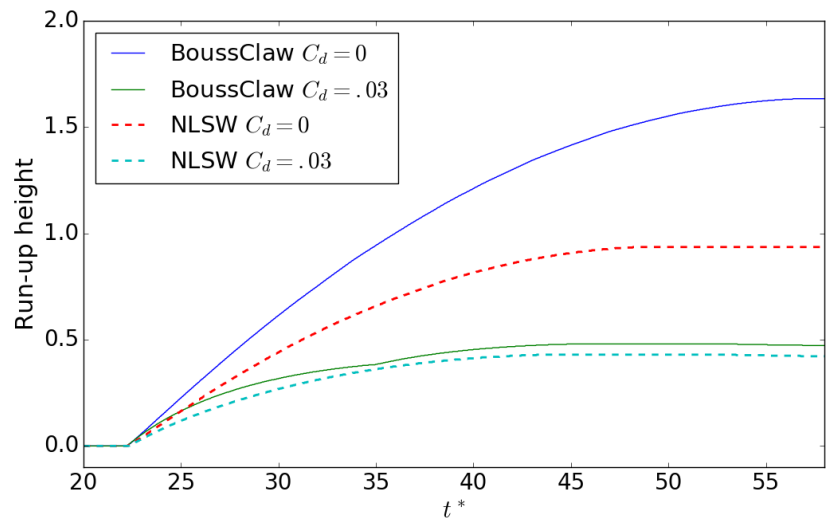

Figure 11: Dimensionless shoreline elevation for the breaking case $(\alpha=0.28$ and a $1: 19.85$ slope) computed by BoussClaw (without $\epsilon_{B}$ ) and NLSW with different $C_{d}^{*}$. 


\begin{tabular}{cccccc}
\hline$C_{d}^{*}$ & 0 & 0.01 & 0.02 & 0.03 & \\
\hline BoussClAw (without $\left.\epsilon_{B}\right)$ & 1.634 & 0.921 & 0.691 & 0.576 & \\
BoussCLAW $\left(\epsilon_{B}=0.8\right)$ & 1.193 & 0.848 & 0.696 & 0.608 & \\
NLSW & 0.936 & 0.702 & 0.596 & 0.528 & \\
\hline Experiment & & & & & 0.551 \\
\hline
\end{tabular}

Table 2: Maximum run-up height, $R^{*}$, for a solitary wave of amplitude $\alpha=0.28$ incident on a $1: 19.85$ slope.

\section{Shoaling and breaking phenomena}

\subsection{Shoaling until breaking}

Wei et al. 1995b made computations of pre-breaking solitary wave shoaling using their fully nonlinear extension of Nwogu's model, a full potential theory, and the weakly nonlinear version of Nwogu's model. They found that the fully nonlinear Boussinesq equations were superior to those of Nwogu in the later stages of the shoaling. In this subsection we will do a similar comparison for our models on the $1: 19.85$ slope which was not included in the reference. Our fully nonlinear Boussinesq model is different from that of the references, as it is a Serre type model with the depth averaged velocity as primary unknown, and our Bouss Claw model is not fully nonlinear. Hence, it is imperative to test the shoaling properties, particularly for the latter model.

We use the set-up described in section 3.3 .2 for the Boussinesq modeling of solitary waves on a slope. The Bouss CLAW simulations are compared with those of other Boussinesq solvers, namely Funwave (Shi et al., 2012), GloBouss (Løvholt et al., 2010) and the Serre type formulation (Løvholt et al., 2013). As noted above, the original Serre's equations are enhanced by adding the same kind of dispersion correction terms as are used in (3).

In Figure 12 surfaces from the different wave models are shown at selected times. The BoussClawmodel is run without the switch to the NLSW equations at $\epsilon_{B}=0.8$ and with the dispersion parameter $B=1 / 15$. However, the results for $B=0$ are rather similar to those for $B=1 / 15$ in this case. At $t^{*}=16$, the 

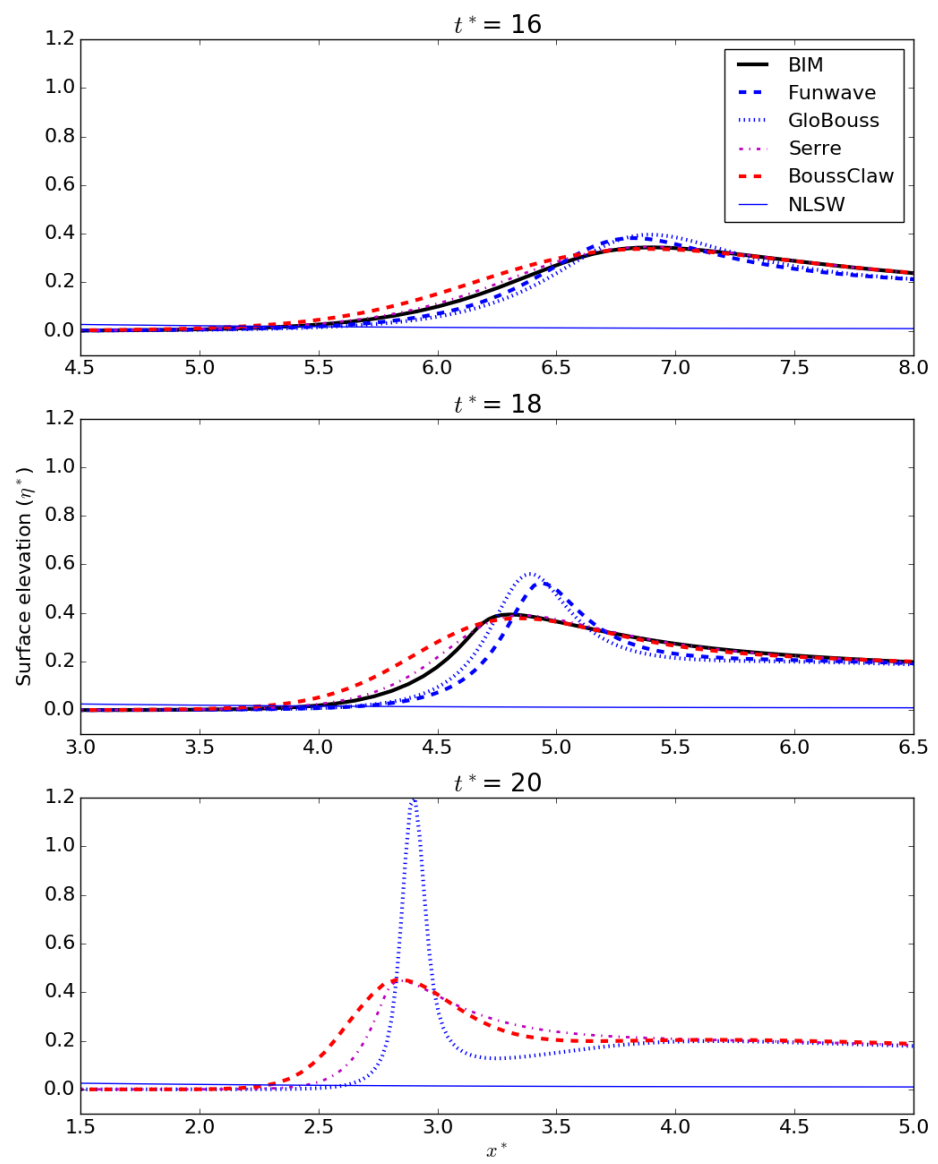

Figure 12: Evolution of $\eta^{*}$ for $\alpha=0.28$ and a $1: 19.85$ slope. Surfaces from the BIM, Serre, GloBouss, BoussClaw and Funwave models at $t^{*}=16,18,20$ are included. The BoussClaw model is used with $\mathrm{B}=1 / 15$, and the Peregrine's equations are used for GloBouss.

computational results from all the Boussinesq-type equations are similar. The NLSW model, on the other hand, yields premature breaking causing a too low amplitude. At $t^{*}=18$, some discrepancies are observed. The models can be split into two groups; GloBouss and Funwave are similar, while the BoussClaw and the Serre results are similar. The wave front from the BoussClaw model 
is somewhat more advanced toward the beach than that from the BIM model. At $t^{*}=20$ the simulation with $\epsilon_{B}=0.8$ has already been in NLSW mode for 5 time units and the difference in the wave height from the full Boussinesq simulation is significant. In fact, the threshold solution is closer to the NLSW solution.

385

At $t^{*}=25$ and $t^{*}=30$, the wave is running up the slope, and the difference in the swash tongue is relatively small.

Other measures of nonlinearity than $\epsilon_{B}$ may be used for model decisions (Lynett, 2006, Matsuyama et al., 2007). Figure 14 shows $\epsilon_{B}, u^{*} / \sqrt{H^{*}}\left(H^{*}\right.$ 


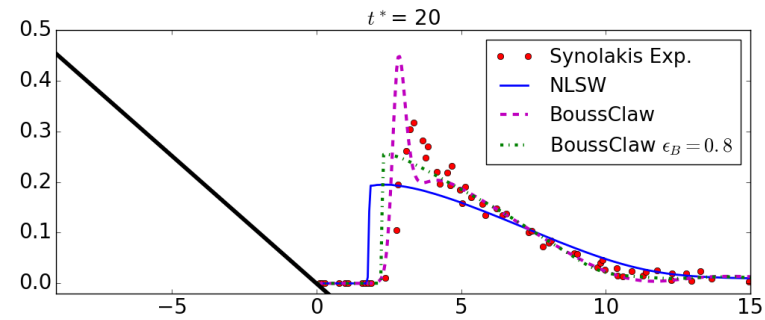

$t^{*}=25$
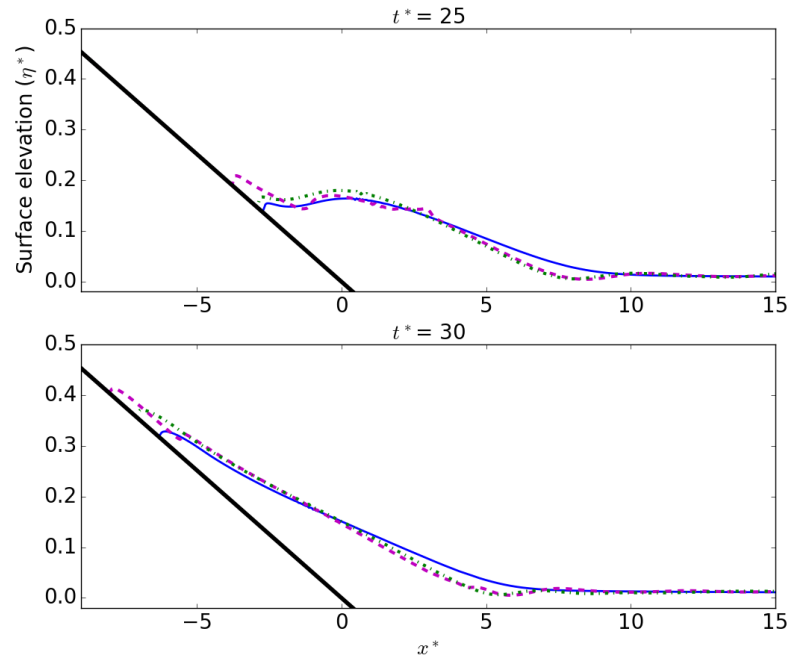

Figure 13: Breaking case ( $\alpha=0.28$ and a $1: 19.85$ slope). Comparison of $\eta^{*}$ from BoussClaw and NLSW with $\epsilon_{B}=0.8$ and $\Delta x^{*}=0.05$ at $t^{*}=20,25$ and 30. Friction forces have been added with $C_{d}^{*}=0.03$ in all simulations.

is the total, dimensionless, flow depth) and the maximum frontal angle as a function of the crest location. When the the BIM model yields a vertical front at $x^{*}=4.09$, we obtain $u^{*} / \sqrt{H^{*}}=1.034$ and the surface slope angle of $39.1^{\circ}$ at the peak. For the present case this might indicate that the value of $u^{*} / \sqrt{H^{*}}$ at the peak surpassing unity or the slope angle surpassing $30^{\circ}$ may be sounder criteria for identifying breaking than $\epsilon_{B}>0.8$. 


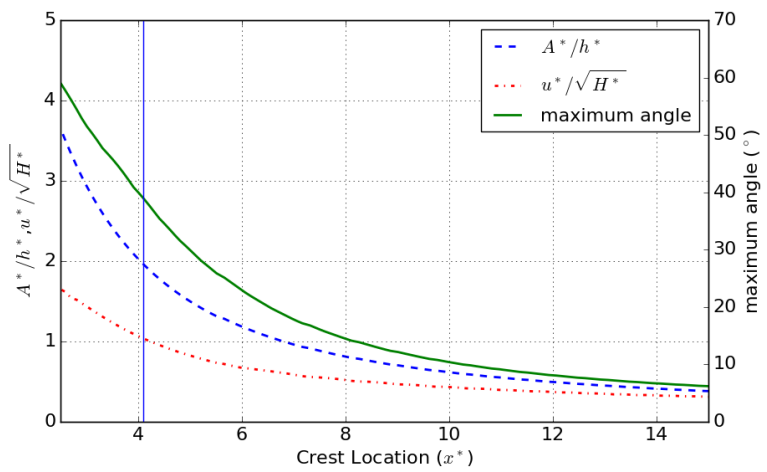

Figure 14: Plot of $A^{*} / h^{*}, u^{*} / \sqrt{H^{*}}$ and maximum angle of waves vs. crest location. The vertical line indicates where the BIM model yields breaking $\left(x^{*}=4.09\right)$.

\subsection{Wave Energy}

The gross wave energies for the shallow water equations and Boussinesq equations are $E_{0}^{*}$ and $E_{0}^{*}+E_{1}^{*}$ respectively, as explained in Appendix B. As stated in section 3.1 they are made dimensionless by the factor $E_{c}=\rho g h_{0}^{3}$. In Figure 15 the energy densities are depicted as functions of the crest location,

$x_{c}^{*}$. In the left panel we observe that the $E_{0}^{*}$ is nearly constant for the shallow water equations until a shock is formed around $x_{c}^{*}=13$. Thereafter, energy is quickly dissipated. For the BoussClaw simulations $E_{0}^{*}$ increases slightly, but noticeably, during shoaling, indicating that $E_{1}^{*}$ needs to be accounted for. In the BoussClaw simulation with no threshold (right panel) $E_{0}^{*}+E_{1}^{*}$ is nearly constant when the wave propagates in constant depth. On the deeper parts of the slope there is first a small increase, then a very moderate reduction. Presumably, the increase is due to the absence of strict energy conservation in the Boussinesq equations. Close to the shoreline this tiny increase is then dominated by a stronger, but still mild, energy dissipation. When the threshold $\epsilon_{B}=0.8$ is invoked there is no difference from the full Boussinesq solution until the threshold is reached for $x_{c}^{*}=x_{B}^{*}=8.03$. After $x_{c}^{*}=x_{B}^{*}$ the hydrostatic energy measure, $E_{0}^{*}$, is the most appropriate for this case. The energy drops momentarily due to the change of energy formula, then remains constant until 
the wave breaks ( $x_{c}^{*}$ around 6$)$, after which a strong dissipation ensues.

In this case the dissipation is due to a single shock. The dissipation rate per width, $D_{t h}$, may then be approximated as (Tissier et al., 2011)

$$
D_{t h}^{*}=\frac{1}{4}\left(\frac{2 h^{*}+d^{*}}{2 h^{*}\left(h^{*}+d^{*}\right)}\right)^{1 / 2}\left(d^{*}\right)^{3},
$$

415 maximum $\eta^{*}\left(A^{*}\right)$ in our case, and $h^{*}$ is the undisturbed water depth. The rate $D_{t h}^{*}$ has been made dimensionless by the factor $E_{c} \sqrt{\frac{g}{h_{0}}}$, where $E_{c}$ is given above. In figure 16 we observe that the dissipation rates of the models has a build-up, before the shock is fully developed, and then agree well with formula (15).

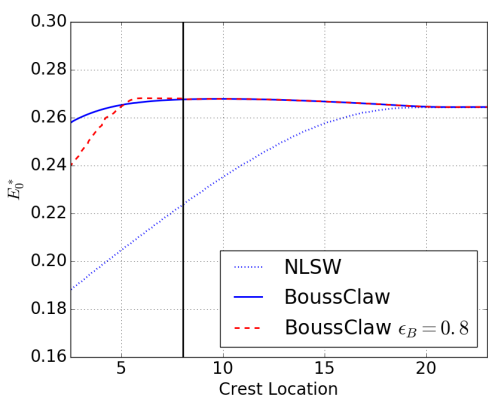

(a)

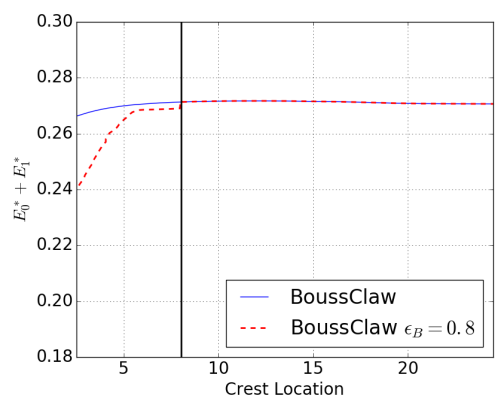

(b)

Figure 15: Wave energy $(\alpha=0.28$, slope $1: 19.85)$ as function of crest position. The vertical line is at $x^{*}=8.03227$ where $\epsilon_{B}=0.8$. (a): $E_{0}^{*}$. (b): Solid line is $E_{0}^{*}+E_{1}^{*}$ of BoussClaw without $\epsilon_{B}$. With $\epsilon_{B}=0.8, E_{0}^{*}$ is shown in dashed line after the threshold is reached.

The onset of the dissipation in the threshold model comes slightly before the a vertical front is observed in the BIM solution $x_{c}^{*}=4.09$. This may point to a too early and strong dissipation. On the other hand, the Boussinesq 


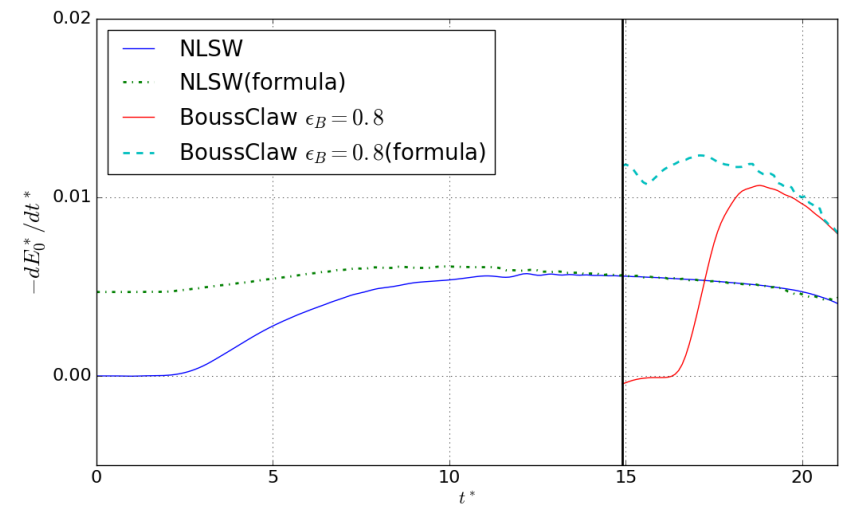

Figure 16: Dimensionless energy dissipation rates for $\alpha=0.28$ and a $1: 19.85$ slope. The label "formula" represents 15 inserted the wave heights from the numerical simulations. BoussClaw with $\epsilon_{B}=0.8$ switches to the NLSW at $t^{*}=14.9$.

solution without the threshold most likely yields too little dissipation. In this context we remark that the experimental data in the upper panel of figure 13 apparently fall between the BoussClaw results with and without the $\epsilon_{B}$ threshold, even though there is scatter in the experimental surface. The last step of the procedure in section 2.1.2, which deals with the dispersion, increases the wave front width to the order of the local depth and thus reduces the dissipation of the next TVD step. This effect of the dispersion terms may be inferred from, for instance, the Green function of Helmholtz type equations as outlined in Glimsdal et al. (2004), section 3.1.1. Still, the BoussCLAW model, without the threshold, is stable during both the last part of shoaling and during runup, even for refined grids. This contrasts the non-dissipative Serre model which may be run beyond $x_{c}^{*}=4$, without the strong artificial amplification of the Peregrine type models, but breaks down when the wave reaches the shoreline (results not shown). Hence, BoussClaw, without the switch to the NLSW equation at $\epsilon_{B}=0.8$, may be a good model for gently spilling breakers. 


\section{Concluding remarks}

445

The BoussClaw extension to the GeOClaw package includes Boussinesq type equations and resembles much used general purpose models such as FUNWAVETVD and Coulwave-TVD, but is based on a different and somewhat simpler set of governing equations, as well as a slightly different numerical scheme. Comparisons with other models as well as experiments are good. Moreover, the model does not display the vulnerability to instabilities for strong nonlinearities in shallow water as is observed for some fully nonlinear Boussinesq models (Løvholt et al., 2013).

The experiments of Synolakis (1987) and a full potential reference model enabled us to assess a set of different long wave models, and BoussCLAw in particular. Using the potential model, we were able to assess in detail the pre-breaking behavior of the models, and to identify the point of breaking accurately. First, we found that by using standard NLSW models, the point of breaking will be located too far offshore and that the resulting dissipation artificially check the amplification. Standard Boussinesq equations, like the so called Peregrine variant, yield marked over-amplification even before the potential theory predicts breaking and they eventually produce completely erroneous wave shape as well as height. The fully nonlinear, non-dissipative model of the Serre type, on the other hand, follows full potential theory very well up to the point of potential-model breaking and avoid severe over-amplification and shape distortion also in the following evolution, until it breaks down at the shoreline. For the pre-breaking part, this is in agreement with earlier investigations of equations of the Nwogu/Wei type (Wei et al., 1995a) and shows that the combined effects of nonlinearities and dispersion influence the solution markedly, when accumulated to the point of breaking. However, herein we find also a very good pre-breaking performance of the BoussCLAw Boussinesq equations where only some nonlinearity is retained in the dispersion. This suggest that the practice of retaining full nonlinearity in Boussinesq shoaling/runup models may be relaxed, especially when the switch to the NLSW equations are invoked. 
This may help reducing stability problems that are observed for fully nonlinear

Boussinesq equations (Løvholt et al., 2013).

A current practice has evolved in which the Boussinesq terms are omitted near shore through the $A / h>0.8$ threshold criterion, inspired by the maximum height of a non-breaking undular bore. In an example presented herein, we investigated the near shore propagation over a relatively gentle shelf of $1: 19.85$ slope, and in this case the actual onset of breaking occurred for $A / h \approx 2$, which is significantly later than what would be predicted by the threshold. Hence, a threshold criterion may lead to an erroneous breaking point as well as an inaccurate description of the later stages of the shoaling. It is noted that the artificial effect discovered would depend on the slope, and a 0.8 threshold may well work better on a much gentler slope as it is primarily derived based on solitary wave properties in constant depth. On the other hand, $1: 19.85$ slope is already quite gentle, and the offset between the reference solution and Boussinesq models using this criteria may be even more pronounced for steeper slopes.

\section{Appendix A. Stability of the hybrid scheme}

It is difficult to analyze the numerical stability for our full Boussinesq equations. To obtain some insight in the stability of the proposed hybrid numerical scheme, we thus consider a closely related, but simpler, equation, namely the linearized Benjamin-Bona-Mahony (BBM) equation (Benjamin et al. (1972))

$$
u_{t}+c u_{x}=\frac{h^{2}}{6} u_{t x x}
$$

where $c=\sqrt{g h}$. This equation describes weakly dispersive, uni-directional waves in constant depth. The equation replaces the momentum equation, whereas no separate continuity equation is involved.

Following the steps of section 2.1.2, we rearrange the equation A.1 as

$$
(I-D)\left(u_{t}+c u_{x}\right)+D u_{x}=0,
$$


where $D=\frac{h^{2}}{6} \partial_{x}^{2}$. The first step of hybrid scheme for this equation is integration of the advection equation

$$
u_{t}+c u_{x}=0
$$

by the finite volume method. Then the Runge-Kutta method is applied to,

$$
(1-D) u_{t}+c D u_{x}=0
$$

which is the counterpart to 8 .

If we use the centered spatial difference approximation of $O\left(\Delta x^{2}\right)$ accuracy on a regular grid we may employ a standard von Neumann analysis where we calculate the growth of an harmonic mode over a single time step. Expressing the coefficients of the velocity array before the time step as $u_{j}=e^{i \xi j \Delta x}$ we then replace the coefficient of $\mathbf{M}^{k}$, defined in section 2.1.2 by $M_{j}^{k}=U_{j}^{k}=g^{k} e^{i \xi j \Delta x}$, where $k$ is $1,2,3,4$ or + . Correspondingly, the coefficients of the $\mathbf{S}^{k}$ array, which contains auxiliary, nodal values for $u_{t}$, is expressed $\left(S_{j}^{k}\right)=s^{k} e^{i \xi j \Delta x}$.

The stability of the first step, A.3, is assured by the standard CFL criterion

$$
\frac{c \Delta t}{\Delta x}<1
$$

If we instead solve the NLSW equations, as in BoussCLAW, $c$ must be replaced by the nonlinear characteristic velocity, which may lead to a more strict criterion. However, the method employed in the first step is not suited for a von Neumann stability analysis and we thus apply this technique to the second step only. Hence, we may put $g^{1}$ to unity, but it is preferable to retain it in the calculations. The Runge-Kutta scheme for time stepping, (9), may now be expressed as

$$
g^{2}=g^{1}+\frac{\Delta t}{2} s^{1}, \quad g^{3}=g^{1}+\frac{\Delta t}{2} s^{2}, \quad g^{4}=g^{1}+\Delta t s^{3},
$$

The discrete version of A.4, which is the counterpart to 10 for the BBM equation reads

$$
S_{j}^{k}-\frac{h^{2}}{6} \frac{S_{j+1}^{k}-2 S_{j}^{k}+S_{j-1}^{k}}{\Delta x^{2}}=-\frac{c h^{2}}{6} \frac{U_{j+2}^{k}-2 U_{j+1}^{k}+2 U_{j-1}^{k}-U_{j-2}^{k}}{2 \Delta x^{3}},
$$


which, inserted the harmonic expressions, implies

$$
s^{k}=i \frac{\gamma}{\Delta t} g^{k}, \quad \gamma=c \Delta t \frac{2 \sin (\xi \Delta x)(1-\cos (\xi \Delta x))}{6 \Delta x^{3} h^{-2}+2 \Delta x(1-\cos (\xi \Delta x))},
$$

where the $\Delta t$ factors are included for convenience. The assembling of the intermediate values in the Runge-Kutta procedure, 11, now yields

$$
g^{+}=g^{1}+\frac{\Delta t}{6}\left[s^{1}+2 s^{2}+2 s^{3}+s^{4}\right] .
$$

By combination of A.5 and A.6 $s^{k}$ and $g^{k}, k=1, \ldots, 4$ can be calculated successively and combined in A.7 to provide the value of $g^{+}$,

$$
\begin{aligned}
g^{+}(\gamma) & =\left(1-\frac{1}{2} \gamma^{2}+\frac{\gamma^{4}}{24}+\left(\frac{\gamma^{3}}{6}-\gamma\right) i\right) g^{1} \\
\left|g^{+}(\gamma)\right|^{2} & =\left(1+\frac{1}{4} \gamma^{4}+\frac{\gamma^{8}}{24^{2}}-\gamma^{2}+\frac{\gamma^{4}}{12}-\frac{\gamma^{6}}{24}+\gamma^{2}+\frac{\gamma^{6}}{36}-\frac{\gamma^{4}}{3}\right)\left|g^{1}\right|^{2} \\
& =\left(1-\frac{1}{72} \gamma^{6}+\frac{1}{576} \gamma^{8}\right)\left|g^{1}\right|^{2} .
\end{aligned}
$$

Stability requires $\left|g^{+}(\gamma) / g^{1}\right|<1$ which is equivalent to $|\gamma|<2 \sqrt{2}$. Moreover, it is easily seen that $\gamma<c \Delta t / \Delta x$. Hence, a sufficient condition for stability of the second step of the hybrid scheme is

$$
\frac{c \Delta t}{\Delta x}<2 \sqrt{2} .
$$

This is more relaxed than the CFL condition for the advection equation A.3. Therefore, if the CFL condition is satisfied in the advection equation, the fractional step is always stable with the suggested numerical scheme.

\section{Appendix B. Energy estimates and dissipation}

\section{Appendix B.1. Velocity field}

To derive the energy estimates for the Boussinesq-type equations, we define the depth-averaged velocity as,

$$
\bar{u}=\frac{1}{H} \int_{-h}^{\eta} u d z
$$


Then the velocity $u$ can be expressed as $u=\bar{u}+u_{1}$ where $u_{1}=O\left(\mu^{2} \bar{u}\right)$ and

$$
\int_{-h}^{\epsilon \eta} u_{1} d z=0
$$

Then the kinematic boundary condition at the bottom and zero divergence implies

$$
w=-h_{x} \bar{u}-\bar{u}_{x}(z+h)+O\left(\mu^{2}\right)
$$

Appendix B.2. Energy integrals

The potential energy density per horizontal area is

$$
V=\int_{-h}^{\eta} \rho g z d z=\frac{1}{2} \rho g \eta^{2}-\frac{1}{2} \rho g h^{2},
$$

In finite depth, the last term, $\frac{1}{2} \rho g h^{2}$, is the equilibrium energy, which is normally excluded from the wave energy. Correspondingly, the first term, which is of order $\epsilon^{2}$ relative to equilibrium energy, is then associated with the wave. However, in the swash zone and during draw-down this distinction is not applicable. Hence, instead of omitting the equilibrium energy locally, we will eventually compute the total energy of the computational domain and then subtract the total, initial equilibrium energy. The kinematic energy density has two contributions,

$$
T=T_{u}+T_{w} ; \quad T_{u}=\frac{\rho}{2} \int_{-h}^{\eta} u^{2} d z, \quad T_{w}=\frac{\rho}{2} \int_{-h}^{\eta} w^{2} d z .
$$

where $T_{w}=O\left(\mu^{2} T_{u}\right)$. For the horizontal part, $T_{u}$ is

$$
T_{u}=\frac{\rho}{2} \int_{-h}^{\eta} u^{2} d z=\frac{\rho}{2} \int_{-h}^{\eta}\left[\bar{u}^{2}+2 \bar{u} u_{1}+u_{1}^{2}\right] d z=\frac{\rho}{2} H \bar{u}^{2}\left(1+O\left(\mu^{4}\right)\right),
$$

since $\bar{u}$ is independent of $z$ and $u_{1}=O\left(\mu^{2} \bar{u}\right)$. The vertical part becomes

$$
\begin{aligned}
T_{w} & =\frac{\rho}{2} \int_{-h}^{\eta}\left[h_{x}^{2} \bar{u}^{2}+2 h_{x} \bar{u} \bar{u}_{x}(z+h)+\bar{u}_{x}^{2}(z+h)^{2}\right] d z \\
& =\frac{\rho}{2} H\left(h_{x}^{2} \bar{u}^{2}+H h_{x} \bar{u} \bar{u}_{x}+\frac{1}{3} H^{2} \bar{u}_{x}^{2}\right),
\end{aligned}
$$

where relative errors of order $\mu^{2}$ are implicit. Thus the energy of a wave can be approximated as

$$
e=\left(e_{0}+e_{1}+O\left(\mu^{4} \epsilon^{2} e_{0}\right)\right)
$$


where $e_{1}=O\left(\mu^{2} \epsilon^{2} e_{0}\right)$ and

$$
\begin{aligned}
e_{0} & =\frac{\rho}{2}\left(g \eta^{2}-g h^{2}+H \bar{u}^{2}\right), \\
e_{1} & =\rho\left(\frac{1}{6} H^{3} \bar{u}_{x}^{2}+\frac{1}{2} H^{2} h_{x} \bar{u} \bar{u}_{x}+\frac{1}{2} H h_{x}^{2} \bar{u}^{2}\right) .
\end{aligned}
$$

We assume a beach to the left, a fixed off-shore boundary of computational domain to the right and an initial wave that does not affect the shoreline. Then, the total wave energies per width are defined as

$$
E_{0}=\int_{x_{a}}^{x_{b}} e_{0} d x-\int_{x_{0}}^{x_{b}}-\frac{1}{2} \rho g h^{2} d x, \quad E_{1}=\int_{x_{a}}^{x_{b}} e_{1} d x,
$$

where $x_{a}$ is the position of the instantaneous shoreline, $x_{0}$ is the position of the equilibrium shoreline, and $x_{b}$ denote off-shore boundary of the computational domain. The latter term is in $E_{0}$ yield subtraction of the initial equilibrium energy. For other geometries, the integration limits must be modified accordingly.

\section{References}

V. V. Titov, C. E. Synolakis, Modeling of breaking and nonbreaking long-wave evolution and runup using VTCS-2, J. Waterw., Port, Coast., Ocean Engrg. 121 (6) (1995) 308-316.

F. Imamura, Long-wave runup models, chapter Simulation of wave-packet propagation along sloping beach by TUNAMI-code, World Scientific 3 (1996) 4.

S. Harig, Chaeroni, W. S. Pranowo, J. Behrens, Tsunami simulations on several scales, Ocean Dynamics 58 (5) (2008) 429-440, ISSN 1616-7228.

M. J. Berger, D. L. George, R. J. LeVeque, K. T. Mandli, The GeoClaw software for depth-averaged flows with adaptive refinement, Adv. Water Res. 34 (2011) $1195-1206$.

D. H. Peregrine, Calculations of the development of an undular bore, J. Fluid Mech. 25 (1966) 321-330. 
Brocchini, A reasoned overview on Boussinesq-type models: the interplay between physics, mathematics and numerics, Proc. R. Soc. 469 (2013) 20130496.

S. Glimsdal, G. Pedersen, C. Harbitz, F. Løvholt, Dispersion of tsunamis: does it really matter?, Nat. Hazards Earth Syst. Sci. 13 (2013) 1507-1526.

F. Løvholt, G. Pedersen, C. Harbitz, S. Glimsdal, J. Kim, On the characteristics of landslide tsunamis, Phil. Trans. R. Soc. A 373 (2053) (2015) 20140376.

J. Grue, E. N. Pelinovsky, D. Fructus, T. Talipova, C. Kharif, Formation of undular bores and solitary waves in the Strait of Malacca caused by the 26 December 2004 Indian Ocean tsunami, J. Geophys. Res. 113 (2008) C05008.

F. Løvholt, G. Pedersen, G. Gisler, Oceanic propagation of a potential tsunami

J. Behrens, F. Dias, New computational methods in tsunami science, Phil. Trans. R. Soc. A 373 (2053) (2015) 20140382.

P. A. Madsen, O. R. Sørensen, A new form of the Boussinesq equations with improved linear dispersion characteristics. Part 2. A slowly-varying bathymetry, Coastal Engineering 18 (3) (1992) 183-204.

O. Nwogu, Alternative form of Boussinesq equations for nearshore wave propagation, J. Waterw., Port, Coast., Ocean Engrg. 119 (6) (1993) 618-638.

D. H. Peregrine, Long waves on a beach, J. Fluid Mech. 27 (04) (1967) 815-827.

P. Madsen, H. Bingham, H. Schäffer, Boussinesq type formulations for fully nonlinear and extremely dispersive water waves: derivation and analysis, Phil. Trans. R. Soc. Lond. A 459 (2003) 1075-1004.

J. T. Kirby, Boussinesq Models and Their Application to Coastal Processes across a Wide Range of Scales, J. Waterw. Port, Coastal, Ocean Eng 142 (6).

H. A. Schäffer, P. A. Madsen, R. Deigaard, A Boussinesq model for waves 
I. A. Svendsen, Mass flux and undertow in a surf zone, Coastal Engineering 8 (4) (1984) 347-365.

M. Tissier, P. Bonneton, F. Marche, F. Chazel, D. Lannes, A new approach to handle wave breaking in fully non-linear Boussinesq models, Coastal Engineering 67 (2012) 54-66.

A. B. Kennedy, Q. Chen, J. T. Kirby, R. A. Dalrymple, Boussinesq modeling of wave transformation, breaking, and run-up. Part I: 1D., J. Waterw., Port, Coast., Ocean Engrg. 126 (1) (2000) 39-47.

P. J. Lynett, Nearshore wave modeling with high-order Boussinesq-type equations, J. Waterw., Port, Coast., Ocean Engrg. 132 (5) (2006) 348-357.

F. Løvholt, P. Lynett, G. K. Pedersen, Simulating run-up on steep slopes with operational Boussinesq models; capabilities, spurious effects and instabilities, Nonlin. Processes Geophys. 20 (2013) 379-395.

M. Matsuyama, M. Ikeno, T. Sakakiyama, T. Takeda, A study of tsunami wave fission in an undistorted experiment, Pure and Applied Geophysics 164 (2-3) (2007) 617-631.

M. Antuono, V. Liapidevskii, M. Brocchini, Dispersive Nonlinear Shallow-Water Equations, Studies in Applied Mathematics 122 (2009) 1-28.

K. Erduran, S. Ilic, V. Kutija, Hybrid finite-volume finite-difference scheme for the solution of Boussinesq equations, Int. J. for Num. Meth. in Fluids 49 (2005) 1213-1232.

D.-H. Kim, P. Lynett, S. Socolofsky, A depth-integrated model for weakly dispersive, turbulent, and rotational flows, Ocean Modelling 27 (2009) 198-214.

J. B. Shiach, C. G. Mingham, A temporally second-order accurate Godunov575 type scheme for solving the extended Boussinesq equations, Coastal Engineering 56 (2009) 3245. 
V. Roeber, K. F. Cheung, M. H. Kobayashi, Shock-capturing Boussinesq-type model for nearshore wave processes, Coastal Engineering 57 (2010) 407423.

D. Dutykh, T. Katsaounis, D. Mitsotakis, Finite volume schemes for dispersive wave propagation and runup, J. Comput. Phys. 230 (2011) 30353061.

F. Shi, J. T. Kirby, J. C. Harris, J. D. Geiman, S. T. Grilli, A high-order adaptive time-stepping TVD solver for Boussinesq modeling of breaking waves and coastal inundation, Ocean Modelling 43 (2012) 36-51.

M. Tonelli, M. Petti, Hybrid finite volume-finite difference scheme for 2DH improved Boussinesq equations, Coastal Engineering 56 (5) (2009) 609-620.

Clawpack Development Team, Clawpack software, URL http://www. clawpack.org, version 5.3.1, 2016.

H. A. Schäffer, P. A. Madsen, Further enhancements of Boussinesq-type equations, Coastal Engineering 26 (1) (1995) 1-14.

J. Kim, Finite volume methods for Tsunamis genereated by submarine landslides, Ph.D. thesis, University of Washington, 2014.

R. J. LeVeque, Wave propagation algorithms for multidimensional hyperbolic systems, J. Comput. Phys. 131 (2) (1997) 327-353.

D. L. George, Augmented Riemann solvers for the shallow water equations over variable topography with steady states and inundation, J. Comput. Phys. 227 (6) (2008) 3089-3113.

M. Tissier, P. Bonneton, F. Marche, F. Chazel, D. Lannes, Serre Green-Naghdi modelling of wave transformation breaking and run-up using a high-order finite-volume finite-difference scheme, Coastal Engineering Proceedings 1 (32) (2011) 13.

D. Dutykh, T. Katsaounis, D. Mitsotakis, Finite volume methods for unidirectional dispersive wave models, International Journal for Numerical Methods in Fluids 71 (6) (2013) 717-736. 
R. J. LeVeque, Finite volume methods for hyperbolic problems, vol. 31, Cambridge university press, 2002.

V. T. Chow, Open channel hydraulics, McGraw-Hill Book Company, Inc; New York, 1959.

Q. Chen, Fully nonlinear Boussinesq-type equations for waves and currents over porous beds, J. of Eng. Mech., ASCE 132 (2) (2006) 220-230.

G. Wei, J. T. Kirby, S. T. Grilli, R. Subramanya, A fully nonlinear Boussinesq model for surface waves. Part 1. Highly nonlinear unsteady waves, J. Fluid Mech. 294 (1995a) 71-92.

M. Briggs, C. Synolakis, U. Kanoglu, D. Green, Runup of solitary waves on a

口 vertical wall, Costal Hydraulics Laboratory, URL http://chl.erdc.usace. army.mil/chl . aspx?p=s\&a=Projects;36, 1995.

C. E. Synolakis, The runup of solitary waves, J. Fluid Mech. 185 (1987) 523-545.

S. Grilli, I. Svendsen, R. Subramanya, Breaking Criterion and Characteristics for Solitary Waves on Slopes, J. Waterw. Port, Coastal, Ocean Eng. 123 (3) (1997) 102-112.

G. K. Pedersen, E. Lindstrøm, A. F. Bertelsen, A. Jensen, D. Laskovski, G. Sælevik, Runup and boundary layers on sloping beaches, Physics of Fluids 25 (2013) pp. 23.

C. Synolakis, E. Bernard, V. Titov, U. Kânoğlu, F. González, Validation and verification of tsunami numerical models, Pure and Applied Geophysics 625 165 (11-12) (2008) 2197-2228.

M. Antuono, L. Soldini, M. Brocchini, On the role of the Chezy frictional term near the shoreline, Theoretical and Computational Fluid Dynamics 26 (1-4) (2012) 105-116. 
G. Wei, J. T. Kirby, S. T. Grilli, R. Subramanya, et al., A fully nonlinear Boussinesq model for surface waves. Part 1. Highly nonlinear unsteady waves, J. Fluid Mech. 294 (7) (1995b) 71-92.

F. Løvholt, G. Pedersen, S. Glimsdal, Coupling of Dispersive Tsunami Propagation and Shallow Water Coastal, Open Oceanography Journal 4 (2010) $71-82$.

S. Glimsdal, G. Pedersen, H. P. Langtangen, An investigation of overlapping domain decomposition methods for one-dimensional dispersive long wave equations, Advances in Water Resources 27 (11) (2004) 1111-1133.

T. B. Benjamin, J. L. Bona, J. J. Mahony, Model equations for long waves in nonlinear dispersive systems, Philosophical Transactions of the Royal Society 640 of London A: Mathematical, Physical and Engineering Sciences 272 (1220) (1972) 47-78. 